\title{
Variables que afectan la relación entre las conductas sustentables y sus consecuencias psicológicas positivas: rasgos de personalidad y costos conductuales
}

\section{Variables Affecting the Relationship Between Sustainable Behaviors and their Positive Psychological Consequences: Personality Traits and Behavioral Costs}

Víctor Corral Verdugo

Karla Robles Pacheco

Nadia Saraí Corral Frías

Universidad de Sonora

\author{
Bernardo Hernández Ruiz \\ Ernesto Suárez \\ Universidad de la Laguna
}

\section{Resumen}

La Conducta Sustentable (CS) puede tener tanto repercusiones psicológicas positivas (bienestar, satisfacción, felicidad) como negativas (costos y sacrificios) en las personas que la practican; sin embargo, no se conoce la causa de esta discrepancia. El presente estudio tuvo como objetivo investigar la posible influencia de variables como los rasgos de personalidad y los costos conductuales en la relación entre CS y sus consecuencias positivas en 100 estudiantes universitarios en una ciudad del norte de México. Se utilizaron instrumentos que registran conductas sustentables, escalas que miden felicidad, bienestar psicológico y satisfacción, así como un inventario de los 5 grandes factores de la personalidad y una escala de costos conductuales del comportamiento sustentable. Se encontraron mayores niveles de beneficios psicológicos que de costos conductuales asociados con las CS. Mediante el empleo de ecuaciones estructurales, el estudio también reveló que los rasgos de personalidad no afectan la relación CS-consecuencias positivas, mientras que los costos conductuales sí la influyen, de manera negativa y en una función híbrida, al fungir tanto como mediadores y como moderadores de la relación.

Palabras clave: mediación, moderación, Conducta Sustentable (CS), costos, beneficios.

Nota del autor

Víctor Corral Verdugo, Departamento de Psicología, Universidad de Sonora (UNISON).

Karla Robles Pacheco, Departamento de Psicología, Universidad de Sonora (UNISON).

Nadia Sarai Corral Frías, Departamento de Psicología, Universidad de Sonora (UNISON).

Bernardo Hernández Ruiz, Facultad de Psicología, Universidad de la Laguna (ULL).

Ernesto Suárez, Facultad de Psicología, Universidad de la Laguna (ULL).

La correspondencia en relación con este artículo debe dirigirse a Víctor Corral Verdugo, Universidad de Sonora, Blvd. Luis Encinas S/N, Col. Centro C.P. 83000, Hermosillo, Sonora, México.

Dirección electrónica: victorcorral@sociales.uson.mx 


\begin{abstract}
Sustainable Behavior (SB) can have both positive (wellbeing, satisfaction, happiness) and negative (costs, sacrifice) psychological impacts on people practicing such behavior; however, the cause of this discrepancy is unknown. This study was aimed at investigating the possible influence of variables such as personality traits and behavioral costs on the relationship between SB and their positive psychological consequences. One hundred college students participated. Instruments assessing $\mathrm{SB}$, scales measuring levels of happiness, psychological wellbeing, and satisfaction, as well as an inventory of the big-five personality factors and a scale assessing behavioral costs were administered. Results indicated higher levels of psychological benefits than behavioral costs associated with SB. By using structural equations, the study also revealed that personality traits do not affect the SBpositive consequences relationship, while behavioral costs do have a negative impact on it, in a hybrid function, serving both as mediators and as moderators of the relationship.
\end{abstract}

Keywords: mediation, moderation, Sustainable Behavior (SB), costs, benefits.

Un buen número de investigaciones en el campo de la psicología ambiental ha mostrado que las CS (comportamiento de cuidado del ambiente físico y social) tienen consecuencias negativas como el malestar y el sacrificio en las personas que las practican (Green-Demers, Pelletier, y Ménard, 1997; Moore, y Boldero, 2017; Steg, Perlaviciute, van der Werff, y Lurvink, 2014). Estas consecuencias pueden explicar la resistencia de muchos individuos a involucrarse en acciones de cuidado ambiental. A pesar de lo anterior, en fechas recientes se ha encontrado que las CS a menudo desembocan en sensaciones psicológicas positivas como el bienestar psicológico, la satisfacción, el reforzamiento positivo intrínseco y la felicidad (Bechtel, y Corral-Verdugo, 2010; CorralVerdugo, Montiel, Sotomayor, Frías, Tapia, y Fraijo, 2011; De Young, 1996; Iwata, 2002;
Moll, Krueger, Zahn, Pardini, Oliveira, y Grafman, 2006).

A pesar de lo aparentemente contradictorio de estos resultados, la evidencia señala que ambos tipos de repercusiones (positivas y negativas) pueden presentarse en las mismas personas. Lo anterior podría implicar que, si las consecuencias positivas predominaran ante las negativas, se facilitaría el despliegue de comportamientos de cuidado ambiental, dado que es mucho más fácil mantener un comportamiento si éste se asocia con un reforzamiento positivo (Corral-Verdugo, González, Rascón, y Corral-Frías, 2016).

Ante la contradicción que arroja el hecho de la aparición concurrente de consecuencias positivas y negativas, el presente estudio pretende responder a las siguientes preguntas: 1. ¿Qué consecuencias predominan en las personas, las negativas o las positivas? y 2 . 
¿Qué variables afectan la relación entre la práctica de CS y sus consecuencias psicológicas positivas? Las respuestas a estas preguntas, en especial a la segunda, son de gran importancia para la psicología ambiental y las ciencias del ambiente en general, ya que al indicar qué es lo que fortalece (o decrementa) la asociación entre las acciones sustentables y sus repercusiones positivas, se puede proveer de una base de conocimiento adecuada para el desarrollo de programas proambientales más efectivos.

\section{Consecuencias negativas de la CS}

Algunas personas reportan como razones para no practicar conductas proambientales los costos que implican tales conductas. La práctica de conductas proambientales se asocia frecuentemente con los sacrificios, la pérdida de tiempo y con el esfuerzo para realizar estas acciones. Dichas conductas son consideradas poco placenteras y su ejecución no se percibe beneficiosa (Moore, y Boldero, 2017), especialmente si se comparan con el desarrollo de conductas antiecológicas, las cuales a menudo producen repercusiones favorables para los individuos (Steg et al, 2014). Los costos percibidos incluyen los sacrificios que deben efectuarse, o los ya realizados, para lograr una meta proambiental. La categoría de costos involucra los recursos económicos, el tiempo, el esfuerzo físico y/o mental que se requieren para desarrollar una conducta (Verhallen, y Pieters,
1984) o las críticas negativas de otras personas (Vanegas-Rico, y Bustos, 2019).

De acuerdo a algunos autores (por ejemplo, Green-Demers et al, 1997), es más probable que una acción se realice si satisface dos criterios: la presencia de altos niveles de autodeterminación y la percepción de bajos costos conductuales. Los bajos costos conductuales representan menores inconvenientes individuales y producen un efecto mínimo en la práctica de conductas. Inversamente, los altos costos reducen la probabilidad de efectuar la conducta, por ende, desembocan en su impedimento o su prohibición. Esto es lo que ha llevado a establecer la llamada Hipótesis del Bajo Costo (Diekmann, y Preisendörfer, 2003). En psicología ambiental se asume que los costos constituyen un factor esencial para explicar la práctica de conductas proambientales (Diekmann, y Preisendörfer, 2003; Moore, y Boldero, 2017; Verhallen, y Pieters, 1984); sin embargo, su estudio es marcadamente escaso. Dentro de las pocas investigaciones desarrolladas, Vanegas-Rico y Corral-Verdugo (2015) crearon y aplicaron una escala de Costos como Requerimientos de Cuidado Ambiental, los cuales se asumieron como dificultades previas a la realización de una conducta proambiental. En un estudio posterior, Vanegas-Rico y Bustos (2019) encontraron una relación negativa entre estos costos y una medida de conducta proambiental (compra de productos verdes). 
Consecuencias positivas de la conducta proambiental

El movimiento de la psicología positiva de la sustentabilidad (Corral-Verdugo, 2012) estudia los factores psicológicos positivos que incitan las CS, entre estos factores se encuentran las consecuencias positivas de estas conductas. La felicidad o bienestar subjetivo se ha señalado como una de esas consecuencias (Bechtel, y Corral-Verdugo, 2010). La felicidad posee, al parecer, una orientación hedónica y otra eudaimónica (Waterman, Schwartz, y Conti, 2008).

La hedonia se identifica con sentirse bien y se define como "maximizar el placer y minimizar el dolor" (Peterson, Park, y Seligman, 2005). La hedonia puede instigar o ser consecuencia de acciones antiambientales (Steg et al., 2014), pero también puede ser una repercusión de conductas sustentables como el altruismo (Moll et al., 2006). La eudaimonia, en contraste, se define como "la búsqueda de una vida significativa" (Peterson et al., 2005, p. 26) y como "intentar usar y desarrollar lo mejor de uno mismo de formas que sean congruentes con los valores propios y con el verdadero yo" (Huta, 2013, p. 140), lo cual sugiere que la eudaimonia orienta al individuo hacia la autorrealización, al desarrollar sus potenciales individuales $\mathrm{y}$ fomentar propósitos de vida (Waterman et al., 2008). La eudaimonia se asocia con la CS (Manríquez, Corral, y Vanegas, 2016) y es afín al bienestar psicológico, el cual se ha reportado como otra consecuencia positiva (Corral-
Verdugo et al., 2011). Otras consecuencias positivas de las acciones sustentables incluyen las sensaciones de satisfacción, autonomía y autoeficacia, que se desprenden de la práctica de esas acciones (Corral-Verdugo et al., 2016; De Young, 1996; Iwata, 2002).

\section{Probables moderadores de la relación CS- consecuencias psicológicas positivas}

$\mathrm{Si}$ se presentan tanto consecuencias negativas como positivas de las CS, ¿Qué factores determinan cuál de estos dos tipos de repercusiones experimentará una persona al practicar CS? Una posible respuesta podría encontrarse en la exploración de las llamadas variables moderadoras y mediadoras. Una variable moderadora es aquella que afecta la dirección o la fuerza de la relación entre una variable independiente y una dependiente (Baron, y Kenny, 1986). En el presente estudio se plantea como hipótesis que variables como la personalidad y los costos conductuales moderarán la relación entre CS y las consecuencias psicológicas positivas de estas conductas (ver figura 1). Otra posible influencia se daría a través de la mediación, en donde la variable mediadora recibiría el efecto de la variable dependiente y afectaría, a su vez, a la independiente (ver figura 2). 


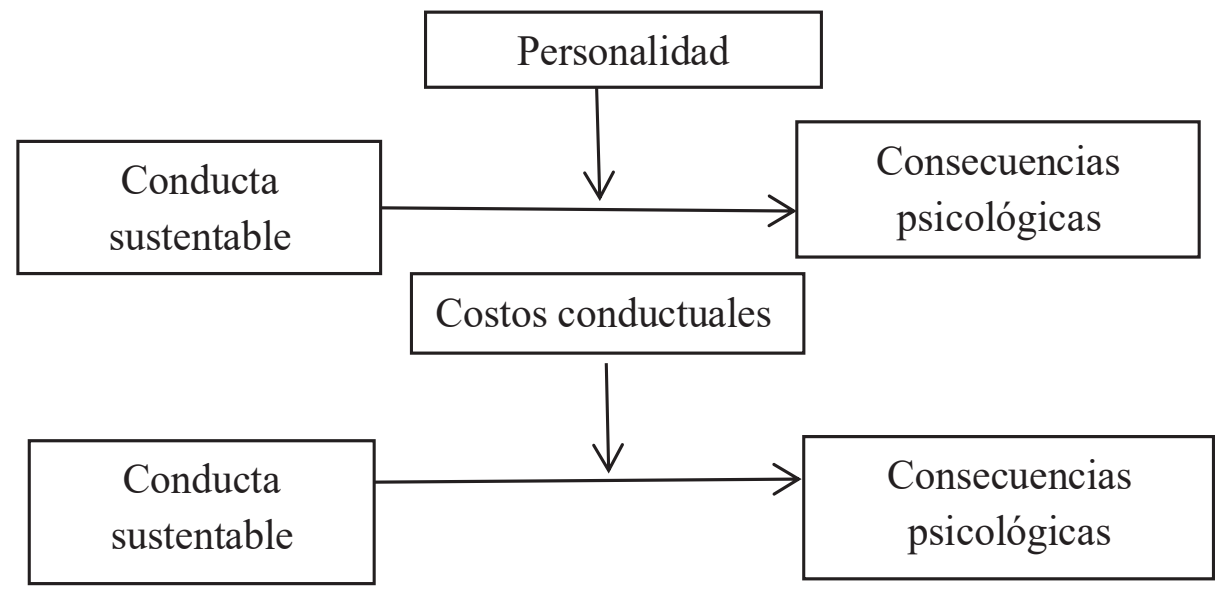

Figura 1. Moderadores personales y de costos de la relación CS-Consecuencias

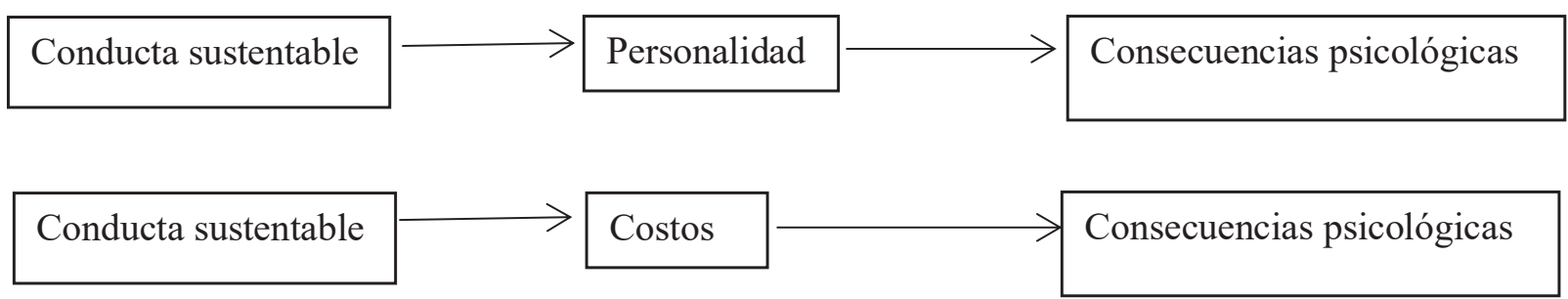

Figura 2. Factores de personalidad y costos como mediadores de la relación CS-Consecuencias

Presumimos que la personalidad puede fungir como moderadora de esa relación, ya que estudios previos han mostrado que rasgos como la amabilidad, la apertura a la experiencia y la extraversión estimulan la práctica de acciones sustentables (Hirsh, 2010; Hirsh y Dolderman, 2007; Markowitz, Goldberg, Ashton, y Lee, 2012). En el caso del neuroticismo, la inestabilidad emocional no anticipa una influencia positiva si lo que se busca es a una persona interesada en el cuidado del ambiente (Toegel, y Barsoux, 2012). Aunque el efecto de los rasgos de personalidad sobre las CS puede ser directo, asimismo, puede especularse que otro componente de ese efecto se da a través de moderar la asociación entre esas conductas y sus consecuencias psicológicas, de manera tal que ciertos tipos de individuos anticipen 
y/o experimenten mayores niveles de molestia o bienestar al practicar acciones sustentables, dependiendo de su personalidad: los más extravertidos, abiertos a la experiencia y amables experimentarían más bienestar, mientras que los más neuróticos tenderían a reportar malestar.

El rol moderador o mediador de los costos es igualmente esperable, aunque su influencia en la relación CS-consecuencias se anticipa será negativa (Diekmann, y Preisendörfer, 2003). Si una persona percibe sacrificio o malestar asociado con sus CS es de esperarse que esa percepción influya en los niveles de beneficios asociados a las CS. A pesar de que esta idea es lógica, no ha sido estudiada, hasta donde se tiene conocimiento, por consiguiente, uno de los propósitos del presente estudio es el de someterla a prueba empírica, junto con la hipótesis de que los rasgos de personalidad influyen en la asociación entre las CS y sus consecuencias psicológicas positivas.

\section{Método}

\section{Participantes}

Participaron 100 estudiantes universitarios (30 hombres y 70 mujeres) de la ciudad de Hermosillo, México, con edades comprendidas entre los 18 y los 28 años, elegidos al azar en las carreras de Educación, Psicología y Biología.

\section{Instrumentos}

Se administró un cuestionario que mide el reporte de CS (proecológicas, frugales, altruistas y equitativas: Tapia, Corral, Fraijo y Durón, 2013). La escala de Conducta Proecológica recoge el reporte de acciones de protección de la naturaleza como cuidar el agua, ahorrar energía y reciclar, entre otras, en una escala de respuesta que va del $0=$ nunca a $3=0$ siempre. La conducta frugal se midió con un instrumento que informa sobre conductas de evitación de desperdicio y derroche de recursos, como reusar productos y vivir sin lujos, el cual se responde en una escala del 0 al 4. El comportamiento altruista fue registrado por medio de una escala en la que se reportan acciones de ayuda desinteresada hacia otras personas, como regalar dinero a necesitados, donar sangre y visitar enfermos; la escala de respuesta va de $0=$ nunca a $3=$ siempre. Para medir la conducta equitativa se empleó un instrumento que recoge el informe de acciones de trato justo y no discriminatorio hacia otros, tales como tratar por igual a pobres y ricos, así como otorgar los mismos derechos a niñas y niños, entre otras, empleando una escala de respuesta del 0 al 4.

Con el fin de medir las consecuencias psicológicas positivas se emplearon escalas que miden felicidad, bienestar psicológico y satisfacción: La escala de Felicidad (Lyubomirsky, y Lepper, 1999) mide de manera global el bienestar subjetivo. Consta de 3 ítems con respuesta tipo Likert del 0 (nada feliz) al 7 (muy feliz). La escala de Bienestar Psicológico (Ryff, 1989), de la que se retomaron 13 ítems indicando autoaceptación, crecimiento personal y propósito con la vida, con un formato de respuesta del $1=$ totalmente en desacuerdo al $6=$ totalmente de acuerdo. La Escala de Satisfacción (Diener, Emmons, Larsen y Griffin, 1985) consta de cinco ítems que evalúan la satisfacción con la 
vida, e incluye reactivos como "estoy satisfecho con mi vida", "si pudiera vivir mi vida otra vez, no cambiaría casi nada", entre otros; el formato de respuesta es de tipo Likert, que va de $1=$ muy en desacuerdo hasta 5= muy de acuerdo. Se utilizó también la escala de los 5 Grandes Factores de Personalidad (Goldberg, 1999), adaptada y traducida para esta investigación (Donnellan,. Oswald, Baird., y Lucas, 2006). La escala es una versión reducida que consta de 20 ítems para medir los cinco rasgos de personalidad (Amabilidad, Responsabilidad, Apertura a nuevas experiencias, Neuroticismo y Extraversión) con un modo de respuesta de escala Likert donde $1=$ fuertemente en desacuerdo hasta $5=$ fuertemente de acuerdo.

Finalmente, los costos conductuales de las CS se midieron empleando parte del instrumento de Vanegas y Bustos (2019) de costos y beneficios de practicar conductas a favor del medio ambiente, seleccionando reactivos de costo como "pierdo tiempo valioso" o "entro en conflicto con familia o pareja" al realizar acciones sustentables. El formato de respuesta va de $1=$ fuertemente en desacuerdo a $5=$ fuertemente de acuerdo. Todos estos instrumentos han mostrado adecuadas propiedades psicométricas de confiabilidad (consistencia interna) y validez convergente y divergente en la población mexicana.

\section{Análisis de datos}

Se efectuaron análisis univariados (medias, desviaciones estándar) para contrastar niveles de las consecuencias (costos, beneficios psicológicos). Dado que existen diferencias entre las escalas de medición para los instrumentos de esas variables, se convirtieron los resultados al estandarizarlos en una escala del 1 al 5, en dos de las variables que no poseían esa escala (Felicidad y Bienestar). Se obtuvieron, además, alfas de Cronbach para estimar la confiabilidad de las escalas utilizadas.

Ya que hay muy pocos estudios que investigan los objetivos de la presente investigación se decidió utilizar un método que analiza si una variable es mediadora o moderadora. El análisis de moderación/ mediación se usó para estimar efectos de las variables ambientales y personales en la relación conductas-consecuencias. Se empleó la estrategia de Sauer y Dick (1993) para probar la hipótesis de una influencia moderadora de las variables de personalidad y costos conductuales (variables de prueba) en la relación entre CS y sus consecuencias positivas. Para lo anterior se utilizaron modelos de ecuaciones estructurales en donde, primeramente, se estimó la relación ( $\beta$ o coeficiente estructural) entre la CS y las consecuencias positivas; posteriormente, en un segundo modelo se estimó la asociación entre la variable supuestamente moderadora o variable de prueba (rasgo de personalidad, costos) y las consecuencias positivas.

Finalmente, se especificó y probó un modelo que incluía las tres variables; en este modelo, la supuesta variable moderadora podría mediar la relación entre la CS y sus consecuencias positivas, estimando también el valor de la asociación directa entre las CS y sus consecuencias positivas. Si el rol mediador de la variable probada (rasgo de personalidad, 
costos) no resultaba significativo $(p>.05)$ y si el valor de la relación directa CS-consecuencias se veía significativamente alterado, se asumía que la variable de prueba funcionaba como una variable puramente moderadora. De manera alternativa, si la asociación directa entre las CS y sus consecuencias positivas se transformaba en no significativa, la variable de prueba no se asumía como moderadora, sino como mediadora. Si tanto la asociación directa como la relación mediada resultaban significativas y el valor de la relación directa se volvía significativamente diferente de cuando el mediador estaba presente, entonces se asumía la ocurrencia de un efecto híbrido moderador-mediador de la variable de prueba (Baron, y Kenny, 1986).

\section{Resultados}

Los análisis univariados de los instrumentos estandarizados en escala de respuestas del 1 al 5 mostraron mayores puntajes en las medias de consecuencias psicológicas positivas (felicidad $=3.96, D E=.86$; satisfacción $=3.85, D E=.82$; bienestar $=3.95, D E=.66)$ que en las de los costos $(2.34, D E=.73)$. Los coeficientes alfa de Cronbach para todos los instrumentos variaron de .63 a .91 (ver tablas de la 1 a la 13).

Tabla 1

Estadísticas univariadas y Alfa de Cronbach para la escala de Conducta Proecológica

\begin{tabular}{|c|c|c|c|c|c|c|}
\hline Ítems & $\mathrm{N}$ & Media & D.E & Máx. & Mín. & Alfa \\
\hline $\begin{array}{l}\text { Espero tener una carga completa de ropa antes de meterla a } \\
\text { la lavadora. }\end{array}$ & 100 & 2.45 & .643 & 3 & 0 & .63 \\
\hline Manejo en vías rápidas a velocidad menor a $60 \mathrm{kph}$. & 88 & .94 & 1.016 & 3 & 0 & \\
\hline Guardo y reciclo el papel usado. & 100 & 1.60 & .921 & 3 & 0 & \\
\hline Separo botellas vacías para reciclar. & 100 & 1.34 & .913 & 3 & 0 & \\
\hline $\begin{array}{l}\text { Le he hecho saber a alguien que se ha comportado de } \\
\text { manera que dañe el ambiente. }\end{array}$ & 100 & 2.17 & .824 & 3 & 0 & \\
\hline Compro comidas preparadas. & 100 & 1.45 & .743 & 3 & 0 & \\
\hline $\begin{array}{l}\text { Compro productos en empaques que pueden volver a uti- } \\
\text { lizarse. }\end{array}$ & 100 & 1.84 & .729 & 3 & 0 & \\
\hline Compro productos (frutas y verduras) de temporada. & 100 & 2.37 & .717 & 3 & 0 & \\
\hline Utilizo la secadora de ropa. & 99 & .75 & 1.037 & 3 & 0 & \\
\hline Leo acerca de temas ambientales. & 100 & 1.37 & .684 & 3 & 0 & \\
\hline $\begin{array}{l}\text { Hablo con amigos de problemas relacionados con el } \\
\text { ambiente. }\end{array}$ & 100 & 1.57 & .830 & 3 & 0 & \\
\hline Mato insectos con un insecticida químico. & 100 & 1.18 & .947 & 3 & 0 & \\
\hline $\begin{array}{l}\text { En el verano apago aire acondicionado cuando dejo mi casa } \\
\text { por más de cuatro horas. }\end{array}$ & 100 & 2.59 & .870 & 3 & 0 & \\
\hline Busco manera de reusar cosas. & 100 & 2.06 & .920 & 3 & 0 & \\
\hline Animo a mis amigos y familiares para que reciclen. & 100 & 1.79 & .954 & 3 & 0 & \\
\hline
\end{tabular}


Tabla 2

Estadísticas univariadas y Alfa de Cronbach para la escala de Altruismo

\begin{tabular}{|c|c|c|c|c|c|c|}
\hline Ítems & $\mathrm{N}$ & Media & D.E & Máx. & Mín. & Alfa \\
\hline $\begin{array}{l}\text { Regalar ropa usada que ya no utiliza pero que está en } \\
\text { buen estado. }\end{array}$ & 100 & 2.02 & .756 & 3 & 0 & .75 \\
\hline $\begin{array}{l}\text { Brindar atención a alguna persona que tropieza, o que } \\
\text { se cae, o que se lastima en la calle. }\end{array}$ & 100 & 2.16 & .792 & 3 & 0 & \\
\hline Contribuir económicamente con la Cruz Roja. & 100 & 1.62 & .738 & 3 & 0 & \\
\hline Visitar a enfermos en hospitales. & 100 & .60 & .684 & 3 & 0 & \\
\hline $\begin{array}{l}\text { Ayudar a personas mayores o incapacitados a cruzar la } \\
\text { calle. }\end{array}$ & 100 & 1.54 & .873 & 3 & 0 & \\
\hline Guiar a personas para localizar alguna dirección. & 100 & 2.23 & .754 & 3 & 0 & \\
\hline Regalar una moneda a indigentes (pobres en la calle). & 100 & 1.85 & .734 & 3 & 0 & \\
\hline Participar en eventos para recolectar fondos. & 99 & 1.11 & .844 & 3 & 0 & \\
\hline $\begin{array}{l}\text { Donar sangre cuando escucha en la radio o televisión } \\
\text { que alguna persona necesita del mismo tipo de sangre } \\
\text { que usted tiene. }\end{array}$ & 100 & .30 & .579 & 3 & 0 & \\
\hline $\begin{array}{l}\text { Colaborar con sus compañeros de escuela o del trabajo } \\
\text { a explicarles y ayudarles en tareas que no entienden. }\end{array}$ & 100 & 2.42 & .716 & 3 & 0 & \\
\hline
\end{tabular}

Tabla 3

Estadísticas univariadas y Alfa de Cronbach para la escala de Frugalidad

\begin{tabular}{|c|c|c|c|c|c|c|}
\hline Ítems & $\mathrm{N}$ & Media & D.E & Máx. & Mín. & Alfa \\
\hline $\begin{array}{l}\text { Si mi carro funciona aún bien, no compro uno más nuevo, } \\
\text { aun teniendo el dinero. }\end{array}$ & 96 & 3.18 & 1.076 & 4 & 0 & .73 \\
\hline $\begin{array}{l}\text { Utilizo la misma ropa que la temporada pasada, aunque } \\
\text { pueda comprarme nueva. }\end{array}$ & 100 & 3.33 & .925 & 4 & 0 & \\
\hline Aun teniendo dinero no compro joyas. & 100 & 3.23 & 1.031 & 4 & 0 & \\
\hline $\begin{array}{l}\text { Me compro muchos zapatos para que combinen con toda } \\
\text { mi ropa. }\end{array}$ & 100 & 2.45 & 1.247 & 4 & 0 & \\
\hline Compro más comida de la necesaria. & 100 & 2.59 & 1.236 & 4 & 0 & \\
\hline Una gran parte de mi dinero la empleo para comprar ropa. & 100 & 2.46 & 1.264 & 4 & 0 & \\
\hline $\begin{array}{l}\text { Casi siempre como en mi casa, en lugar de ir a restau- } \\
\text { rantes o taquerías. }\end{array}$ & 100 & 3.31 & .966 & 4 & 0 & \\
\hline $\begin{array}{l}\text { Si voy a un lugar que no está lejos, prefiero caminar que } \\
\text { mover mi carro. }\end{array}$ & 99 & 2.90 & 1.252 & 4 & 0 & \\
\hline $\begin{array}{l}\text { Reúso los cuadernos y las hojas de papel que sobran al } \\
\text { terminar cada ciclo escolar. }\end{array}$ & 100 & 3.11 & 1.150 & 4 & 0 & \\
\hline Vivo sin lujos, aunque pueda dármelos. & 100 & 2.77 & 1.128 & 4 & 0 & \\
\hline
\end{tabular}


Tabla 4

Estadísticas univariadas y Alfa de Cronbach para la escala de Equidad

\begin{tabular}{lcccccc}
\hline \multicolumn{1}{c}{ Ítems } & $\mathrm{N}$ & Media & D.E & Máx. & Mín. & Alfa \\
\hline $\begin{array}{l}\text { Mi pareja tiene el mismo derecho que yo a decidir sobre } \\
\text { los gastos en la familia. }\end{array}$ & 100 & 3.78 & .579 & 4 & 0 & .61 \\
$\begin{array}{l}\text { En mi trabajo, trato a todos mis compañeros como mis } \\
\text { iguales, sin importar si son o no mis subalternos. }\end{array}$ & 100 & 3.63 & .691 & 4 & 0 \\
$\begin{array}{l}\text { En mi casa, los niños tienen el mismo derecho que los } \\
\text { adultos a tomar decisiones importantes. }\end{array}$ & 100 & 2.85 & .999 & 4 & 0 \\
$\begin{array}{l}\text { Trato a los indígenas de la misma manera que a las } \\
\text { personas que no lo son. }\end{array}$ & 100 & 3.72 & .637 & 4 & 0 \\
$\begin{array}{l}\text { Mi trato para las personas pobres es igual que el que } \\
\text { tengo con los más ricos. }\end{array}$ & 100 & 3.80 & .569 & 4 & 0 \\
$\begin{array}{l}\text { En mi familia, las niñas tienen la misma oportunidad de } \\
\text { estudiar (hasta donde quieran) que los niños. }\end{array}$ & 100 & 3.97 & .171 & 4 & 0
\end{tabular}

Tabla 5

Estadísticas univariadas y Alfa de Cronbach para la escala de Felicidad

\begin{tabular}{|c|c|c|c|c|c|c|}
\hline Ítems & $\mathrm{N}$ & Media & D.E & Máx. & Mín. & Alfa \\
\hline Generalmente me considero... & 100 & 5.56 & 1.290 & 7 & 1 & .87 \\
\hline $\begin{array}{l}\text { Comparado(a) con la mayoría de mis conocidos, yo } \\
\text { me considero... }\end{array}$ & 100 & 5.52 & 1.389 & 7 & 1 & \\
\hline $\begin{array}{l}\text { Soy feliz en lo general; disfruto la vida a pesar de lo } \\
\text { que pase, sacando lo mejor de todas las cosas. }\end{array}$ & 100 & 5.57 & 1.387 & 7 & 1 & \\
\hline
\end{tabular}

Tabla 6

Estadísticas univariadas y Alfa de Cronbach para la escala de Satisfacción

\begin{tabular}{|c|c|c|c|c|c|c|}
\hline Ítems & $\mathrm{N}$ & Media & D.E & Máx. & Mín. & Alfa \\
\hline $\begin{array}{l}\text { En la mayoría de los aspectos, mi vida es como } \\
\text { yo quiero que sea. }\end{array}$ & 100 & 3.63 & .960 & 5 & 1 & .85 \\
\hline Las circunstancias de mi vida son muy buenas. & 100 & 3.95 & .903 & 5 & 1 & \\
\hline Estoy satisfecho con mi vida. & 100 & 4.05 & 1.009 & 5 & 1 & \\
\hline $\begin{array}{l}\text { Hasta ahora he conseguido de la vida las cosas } \\
\text { que considero importantes. }\end{array}$ & 100 & 4.07 & 1.018 & 5 & 1 & \\
\hline $\begin{array}{l}\text { Si pudiera vivir mi vida otra vez, no cambiaría } \\
\text { casi nada. }\end{array}$ & 100 & 3.59 & 1.288 & 5 & 1 & \\
\hline
\end{tabular}


Tabla 7

Estadísticas univariadas y Alfa de Cronbach para la escala de Bienestar Psicológico

\begin{tabular}{|c|c|c|c|c|c|c|}
\hline Ítems & $\mathrm{N}$ & Media & D.E & Máx. & Mín. & Alfa \\
\hline En general, me siento seguro y positivo conmigo mismo. & 100 & 4.51 & 1.210 & 6 & 1 & .91 \\
\hline $\begin{array}{l}\text { He sido capaz de construir un hogar y un modo de vida a mi } \\
\text { gusto. }\end{array}$ & 100 & 3.92 & 1.261 & 6 & 1 & \\
\hline Siento que mis amistades me aportan muchas cosas. & 100 & 4.70 & 1.176 & 6 & 1 & \\
\hline $\begin{array}{l}\text { Me siento bien cuando pienso en lo que he hecho en el } \\
\text { pasado y lo que espero hacer en el futuro. }\end{array}$ & 100 & 4.40 & 1.128 & 6 & 1 & \\
\hline $\begin{array}{l}\text { Mis objetivos en la vida han sido más una fuente de satisfac- } \\
\text { ción que de frustración. }\end{array}$ & 100 & 4.46 & 1.184 & 6 & 1 & \\
\hline Me gusta la mayor parte de los aspectos de mi personalidad. & 100 & 4.65 & 1.114 & 6 & 1 & \\
\hline $\begin{array}{l}\text { Tengo confianza en mis opiniones incluso si son contrarias a } \\
\text { lo que piensa la gente. }\end{array}$ & 100 & 4.64 & 1.243 & 6 & 1 & \\
\hline Tengo clara la dirección y el objetivo de mi vida. & 100 & 4.56 & 1.336 & 6 & 1 & \\
\hline $\begin{array}{l}\text { En general, con el tiempo siento que sigo aprendiendo más } \\
\text { sobre mí mismo. }\end{array}$ & 100 & 5.28 & .933 & 6 & 1 & \\
\hline $\begin{array}{l}\text { En su mayor parte, me siento orgulloso de quién soy y la } \\
\text { vida que llevo. }\end{array}$ & 100 & 4.83 & 1.092 & 6 & 1 & \\
\hline $\begin{array}{l}\text { Sé que puedo confiar en mis amigos, y ellos saben que } \\
\text { pueden confiar en mí. }\end{array}$ & 100 & 5.06 & 1.127 & 6 & 1 & \\
\hline $\begin{array}{l}\text { Tengo la sensación de que con el tiempo me he desarrollado } \\
\text { mucho como persona. }\end{array}$ & 100 & 5.26 & .928 & 6 & 1 & \\
\hline $\begin{array}{l}\text { Para mí, la vida ha sido un proceso continuo de estudio, } \\
\text { cambio y crecimiento. }\end{array}$ & 100 & 5.46 & .937 & 6 & 1 & \\
\hline
\end{tabular}

Tabla 8

Estadísticas univariadas y Alfa de Cronbach para la escala de Costos

\begin{tabular}{lllllcc}
\hline \multicolumn{1}{c}{ Ítems } & $\mathrm{N}$ & Media & D.E & Máx. & Mín. & Alfa \\
\hline Entro en conflicto con mi familia o pareja. & 94 & 2.20 & 1.325 & 5 & 1 & .80 \\
Tengo que renunciar a cosas que me gustan. & 94 & 2.22 & 1.220 & 5 & 1 & \\
Me ven raro. & 94 & 2.17 & 1.206 & 5 & 1 & \\
Empiezo a vivir preocupado(a) todo el tiempo. & 94 & 2.60 & 1.417 & 5 & 1 & \\
Pierdo tiempo valioso haciéndolas. & 94 & 2.34 & 1.169 & 5 & 1 & \\
Tengo que dejar de hacer actividades que común- & 94 & 2.79 & 1.243 & 5 & 1 \\
mente hago. & 94 & 2.11 & 1.274 & 5 & 1 \\
Algunas personas me hacen menos. & 94 & 2.23 & 1.239 & 5 & 1 \\
Las personas podrían pensar que soy pobre. & 94 & 2.37 & 1.218 & 5 & 1 & \\
Me sienta limitado (a). & & & &
\end{tabular}


Tabla 9

Estadísticas univariadas y Alfa de Cronbach para la escala de Extraversión

\begin{tabular}{lcrrrrc}
\hline \multicolumn{1}{c}{ Ítems } & $\mathrm{N}$ & Media & D.E & Máx. & Mín. & Alfa \\
\hline Soy el alma de la fiesta. & 100 & 2.69 & 1.226 & 5 & 1 & .82 \\
No hablo mucho. & 100 & 3.39 & 1.420 & 5 & 1 & \\
Hablo con diferentes personas en fiestas. & 100 & 3.58 & 1.262 & 5 & 1 & \\
Me mantengo a la distancia. & 99 & 3.27 & 1.260 & 5 & 1 & \\
\hline
\end{tabular}

Tabla 10

Estadísticas univariadas y Alfa de Cronbach para la escala de Amabilidad

\begin{tabular}{lcrrrrc}
\hline \multicolumn{1}{c}{ Ítems } & $\mathrm{N}$ & Media & D.E & Máx. & Mín. & Alfa \\
\hline Simpatizo con los sentimientos de los demás. & 100 & 4.07 & 1.066 & 5 & 1 & .61 \\
No estoy interesado en los problemas de otros. & 100 & 3.64 & 1.360 & 5 & 1 & \\
Siento las emociones de los demás. & 100 & 3.77 & 1.153 & 5 & 1 & \\
No estoy realmente interesado en otros. & 100 & 3.88 & 1.183 & 5 & 1 & \\
\hline
\end{tabular}

Tabla 11

Estadisticas univariadas y Alfa de Cronbach para la escala de Responsabilidad

\begin{tabular}{lrrrrrr}
\hline \multicolumn{1}{c}{ Ítems } & $\mathrm{N}$ & Media & D.E & Máx. & Mín. & Alfa \\
\hline Con frecuencia olvido poner las cosas en su lugar. & 100 & 2.68 & 1.254 & 5 & 1 & .61 \\
Me gusta el orden. & 100 & 4.25 & .903 & 5 & 1 & \\
Soy un desastre con mis cosas. & 100 & 3.68 & 1.278 & 5 & 1 \\
\hline
\end{tabular}

Tabla 12

Estadísticas univariadas y Alfa de Cronbach para la escala de Neuroticismo

\begin{tabular}{lrrrrrr}
\hline \multicolumn{1}{c}{ Ítems } & $\mathrm{N}$ & Media & D.E & Máx. & Mín. & Alfa \\
\hline Tengo cambios de humor frecuentemente. & 100 & 2.98 & 1.356 & 5 & 1 & .63 \\
Soy relajado la mayor parte del tiempo. & 100 & 2.75 & 1.258 & 5 & 1 & \\
Me enojo con facilidad. & 100 & 3.07 & 1.402 & 5 & 1 & \\
Raramente me siento triste. & 100 & 3.03 & 1.235 & 5 & 1 & \\
\hline
\end{tabular}


Tabla 13

Estadísticas univariadas y Alfa de Cronbach para la escala de Apertura

\begin{tabular}{lrrrrrc}
\hline \multicolumn{1}{c}{ İ́ms } & $\mathrm{N}$ & Media & D.E & Máx. & Mín. & Alfa \\
\hline Tengo una buena imaginación. & 100 & 3.74 & 1.148 & 5 & 1 & .60 \\
No me interesan las ideas abstractas. & 100 & 3.58 & 1.031 & 5 & 1 & \\
No tengo buena imaginación. & 100 & 4.00 & 1.050 & 5 & 1 & \\
Tengo dificultada para entender ideas abstractas. & 99 & 3.35 & 1.181 & 5 & 1 & \\
\hline
\end{tabular}

Al analizar las posibles influencias moderadoras de los rasgos de personalidad se obtuvieron resultados que no respaldaban las hipótesis de investigación. De acuerdo con la figura 3 , la CS se asocia alta $(\beta=.60)$ y significativamente $(p<.05)$ con las consecuencias psicológicas positivas, en tanto, la figura 4 muestra un coeficiente estructural positivo y significativo de $\beta=.33(p<.05)$ que parte del neuroticismo hacia la CS. Esta última variable no se asoció significativamente con las consecuencias psicológicas positivas (figura5).

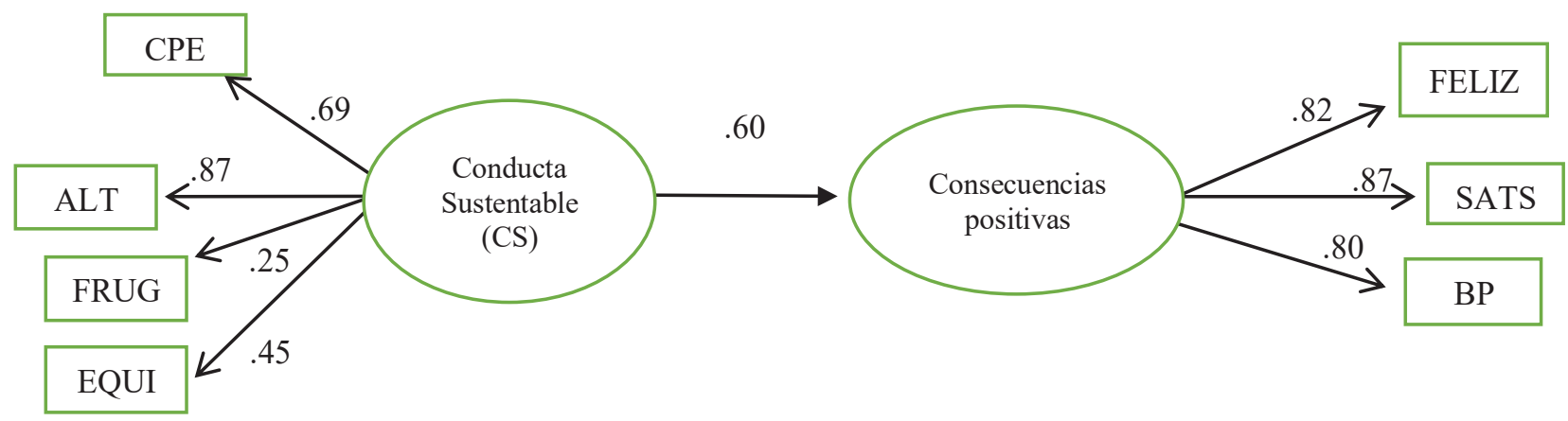

Figura 3. Relación entre las CS y sus consecuencias positivas. Todas las cargas factoriales son significativas $(p<0.05)$. Índices de bondad de ajuste: $X^{2}=24.45(12 g l), p=.10, B N N F I=.90, C F I=.94, R M S E A=.10$

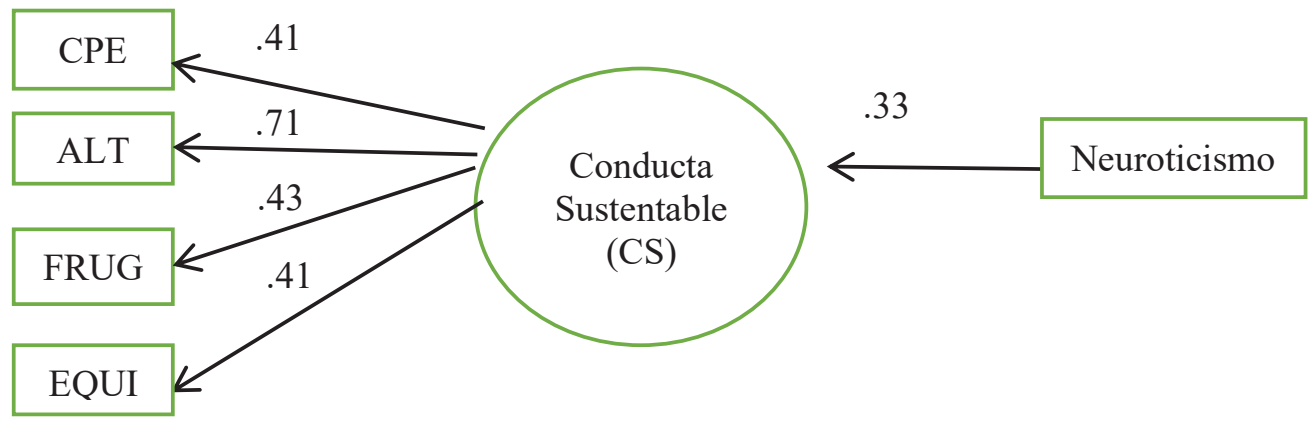

Figura 4. Relación entre las CS y el neuroticismo. Todas las cargas factoriales son significativas $(p<0.05)$. Índices de bondad de ajuste: $X^{2}=1.62,(3 g l) p=.65, B N N F I=1.10, C F I=1.00, R M S E A=.000$ 


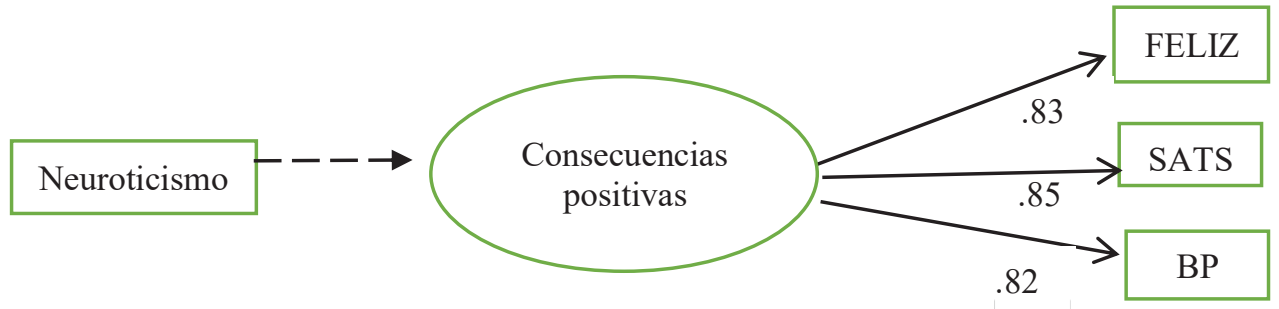

Figura 5. Ausencia de relación entre el neuroticismo y las consecuencias positivas. Todas las cargas factoriales son significativas $(p<0.05)$. Índices de bondad de ajuste: $X^{2}=.13,(2 g l) p=.52, B N N F I=1.00, C F I=1.00, R M S E A=.000$

Con el resto de las variables de personalidad ocurrió algo semejante a lo obtenido con el neuroticismo. Sólo se reportarán los valores de las relaciones entre las CS y cada rasgo de personalidad, entre éstos y las consecuencias positivas y el valor de la relación directa al introducir cada rasgo de personalidad al modelo como posible moderador. La extraversión se asoció positiva y significativamente con las CS $(\beta=.28, p<.05)$, pero no con las consecuencias positivas. En el modelo de moderación, la extraversión no modificó la relación CSconsecuencias positivas, la cual produjo un valor de $\beta=.58(p<.05)$. La responsabilidad se asoció significativamente con las $\operatorname{CS}(\beta=.33$, $p<.05)$ e influyó positiva y significativamente en las consecuencias positivas $(\beta=.32, p<.05)$; sin embargo, no afectó la relación directa entre CS y esas consecuencias positivas (el valor se mantuvo en $\beta=.57)$. En cuanto a la amabilidad, ésta no se relacionó significativamente $(p<.05)$ ni con las CS, ni con las consecuencias positivas, de manera separada; cuando ingresaron todas las variables al modelo de moderación, el valor de la relación directa CS-consecuencias positivas no se modificó $(\beta=.59, p<.05)$. De manera similar, la apertura no se asoció ni con la CS, ni con las consecuencias positivas, de manera independiente y en el modelo de moderación, aunque la relación entre CS y apertura fue significativa y negativa $(\beta=-.23$, $p<.05$ ), la asociación con las consecuencias positivas se mantuvo no significativa y no se encontró modificación a la relación directa CSconsecuencias positivas $(\beta=.61, p<.05)$.

Un panorama distinto surgió del análisis del rol mediador/moderador de la variable costos conductuales. Como se expuso anteriormente, y se observa en la figura 3, la CS influyó notoria $(\beta=.60)$ y significativamente $(p<.05)$ en las consecuencias positivas de esa conducta, en ausencia de cualquier variable de prueba, incluyendo la variable costos conductuales. No se encontró lo mismo para la relación entre las CS y los costos conductuales, la cual resultó no significativa (ver figura 6). Los 
costos, sin embargo, tuvieron un efecto directo moderado y significativo $(\beta=.43, p<.05)$ en las consecuencias psicológicas positivas (ver figura 7). Cuando se integraron las tres variables, los resultados anteriores se vieron alterados en el modelo final, como lo muestra la figura 8 . Dado que tanto la asociación directa $(\beta=.43$, $p<.05)$ como la relación mediada $(\beta=-.36, \beta=$ $-.27, p<.05)$ son significativas en este modelo, además de que el valor de la relación directa decrece de manera notoria (de $\beta=.60$ a $\beta=$ .43) de cuando la variable de prueba estaba ausente a cuando se introdujo en el modelo, se concluye que los costos conductuales influyen en la relación CS-consecuencias positivas. Esta influencia es híbrida, es decir, tanto mediadora como moderadora.

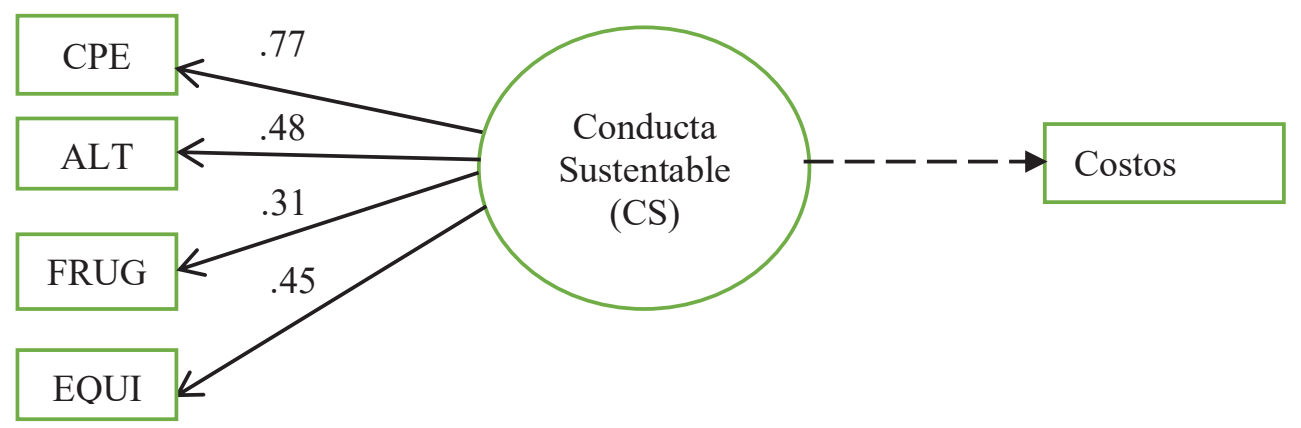

Figura 6. Relación entre las CS y sus costos. Todas las cargas factoriales son significativas $(p<0.05)$. Índices de bondad de ajuste $\left[X^{2}=4.43,(2 g l) p=.10, B N N F I=.93, C F I=.95, R M S E A=.11\right]$

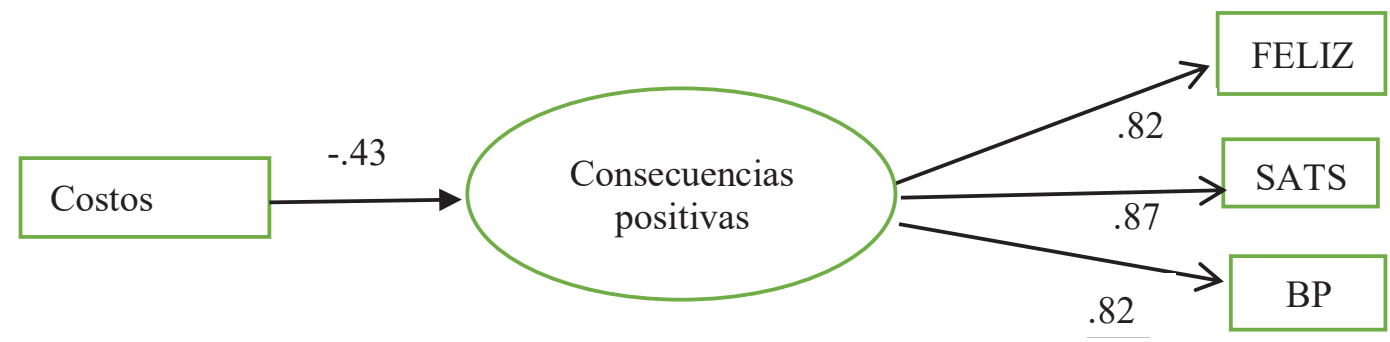

Figura 7. Relación entre los costos y las consecuencias positivas de la CS. Todas las cargas factoriales son significativas $(p<0.05)$, lo mismo que el coeficiente estructural. Índices de bondad de ajuste $\left[X^{2}=4.45,(2 g l), p=.10, B N N F I=.95\right.$, $C F I=.98, R M S E A=.11]$ 


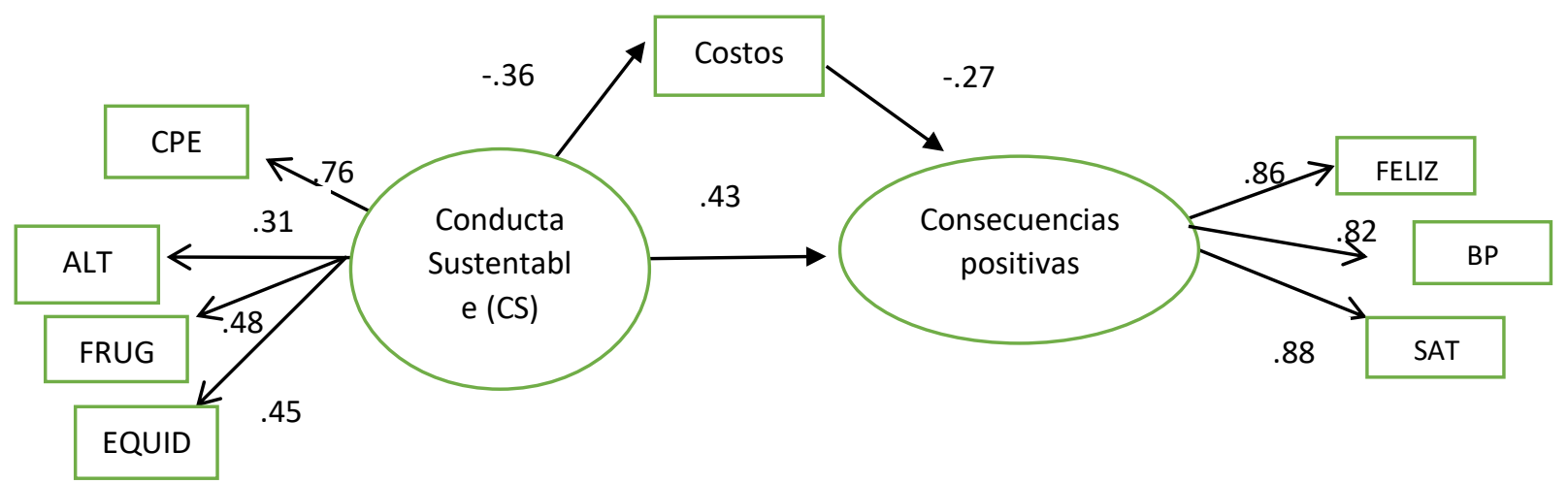

Figura 8. Modelo de moderación/mediación de las CS, Consecuencias Positivas y Costos. Todas las cargas factoriales son significativas $(p<0.05)$. Índices de bondad de ajuste $\left[X^{2}=39.30(16 g l) \mathrm{p}=.001 ., B N N F I=.83, C F I=.91, R M S E A=.12\right]$

\section{Conclusiones}

Al realizar la presente investigación se concluye, con base en los objetivos, que: Los resultados del estudio aquí reportados sugieren que algunos de los rasgos de personalidad, específicamente neuroticismo, extraversión, y responsabilidad, se asocian significativamente, aunque de forma sutil, con la CS. Es de llamar la atención que estos resultados difieren de los hallazgos de estudios previos (Hirsh, 2010; Markowitz et al., 2012), los cuales reportan que la amabilidad y la apertura son rasgos asociados con conductas protectoras del ambiente, mas no así los tres rasgos que aquí resultaron significativamente asociados con las CS.

Los rasgos de personalidad también estuvieron relacionados con consecuencias psicológicas positivas, donde la responsabilidad se relacionó positivamente con estas consecuencias psicológicas. En este caso, no se detectaron estudios previos que permitan contrastar estos hallazgos. No obstante, ninguno de los cinco rasgos investigados modificó la relación entre las CS y sus consecuencias psicológicas positivas, lo que preliminarmente parece descartar su rol moderador.

Cuando se probó el posible efecto de los costos conductuales en la relación CSconsecuencias positivas, los resultados fueron diferentes. Los costos modificaron el valor de la relación directa entre esas dos variables. Además, en el modelo de moderación/mediación las relaciones de los costos conductuales con las CS y las consecuencias positivas fueron de valor negativo y significativas (-.36 y $-.27, p>.05$, respectivamente). Estos resultados sugieren que los costos conductuales disminuyen la fuerza de la asociación entre la CS y sus 
consecuencias positivas. Esto significa que las personas que perciben más esfuerzo, sacrificio e inconveniencia en las conductas de protección ambiental, tenderán a experimentar menores niveles de beneficios psicológicos como consecuencias de actuar cuidando el ambiente físico y social. Estos resultados apoyan la hipótesis del bajo costo y la idea de que los costos constituyen un factor esencial para explicar la práctica de conductas proambientales (Diekmann, y Preisendörfer, 2003; Moore, y Boldero, 2017; Verhallen, y Pieters, 1984). El estudio aquí reportado también parece indicar que las personas experimentan más beneficios psicológicos que costos conductuales asociados con la práctica de CS. Este hallazgo, de replicarse en futuros estudios, ofrecería un punto de partida favorable para intervenciones en las cuales se enfatice la experiencia de estados psicológicos positivos y se busquen minimizar/bloquear la percepción de costos, especialmente si, como lo señalan los resultados de este estudio, los costos afectan la influencia de la CS en los beneficios psicológicos que ésta promueve.

Es importante mencionar que la presente investigación, como todas, tiene limitaciones. Por ejemplo, la muestra es pequeña $(n=100)$ y está conformada sólo por estudiantes universitarios. Por ende, no se sabe si los resultados son generalizables a toda la población. Por esta razón $\mathrm{y}$ dado que las investigaciones antecedentes son escasas, es necesario replicarlas para llegar a conclusiones firmes respecto de la relación entre CS y la personalidad.
A pesar del resultado positivo aquí reportado, es conveniente mencionar que el presente estudió sólo investigó el posible rol mediador/ moderador que dos factores pudieran tener en la relación CS-consecuencias positivas. Es necesario involucrar variables adicionales como los factores ambientales y otras variables psicológicas. Por ejemplo, en contextos donde existen facilidades físicas y modelos/instigadores sociales de las CS es posible observar un mayor despliegue de acciones sustentables. La infraestructura física que afecta las opciones para viajar utilizando transporte público en lugar del privado, la eficiencia energética de las casas, las opciones proambientales que ofrecen los proveedores de bienes y servicios, también afectan las decisiones de practicar CS (Brown, y Werner, 2012; Corral, Caso, y Frías, 2017; Swim, Clayton, y Howard, 2011). Es necesario investigar el rol moderador de estas variables, además de otras de naturaleza psicológica/ disposicional como los valores, las creencias y los motivos proambientales. También es necesario desarrollar este tipo de estudios en población general y en escenarios culturales diversos, de manera que los resultados puedan generalizarse hacia estratos sociales más amplios.

\section{Referencias}

Baron, R., y Kenny, D. (1986). The moderatormediator variable distinction in social psychological research: conceptual, strategic, and statistical considerations. Journal of 
Personality and Social Psychology, 51, 1173 1182.

Bechtel, R., y Corral-Verdugo, V. (2010). Happiness and sustainable behavior. En V. Corral-Verdugo, C. García-Cadena, y M. Frías-Armenta (Eds.), Psychological approaches to sustainability. Current trends in theory, research and applications (pp. 433-450). Nova Science Publishers.

Brown, B. B., y Werner, C. M. (2012). Healthy physical environment and eating: Environmental supports for health. En S. Clayton (Ed.), The Oxford Handbook of Environmental and Conservation Psychology (pp 459-484). Oxford Press.

Corral, V. (2012). The positive Psychology of Sustainability. Environment, Development and Sustainability, 14, 651-666.

Corral, V., Caso, J., y Frías, M. (2017). Psicología del Cambio Climático. Pearson.

Corral, V., González, D., Rascón, M., y Corral-Frías, V. (2016). Intrinsic motives of autonomy, self efficacy, and satisfaction associated with two instances of sustainable behavior: frugality and equity. Psychology, 7, 662-671.

Corral, V., Montiel, M., Sotomayor, M., Frías, M., Tapia, C., y Fraijo, B. (2011). Psychological wellbeing as correlate of sustainable behaviors. International Journal of Hispanic psychology, 4, 31-44.

De Young, R. (1996). Some Psychological Aspects of a Reduced Consumption Lifestyle: The Role of Intrinsic Satisfaction and Competence Motivation. Environment y Behavior, 28, 358-409.

Diekmann, A., y Preisendörfer, P. (2003). Green and greenback: The behavioral effects of environmental attitudes in low-cost and highcost situations. Rationality and Society, 15, 441-472.

Diener, E., Emmons, R., Larsen, R., y Griffin, S. (1985). The satisfaction with life scale. Journal of Personality Assessment, 49, 7175.

Donnellan, M. B., Oswald, F. L., Baird, B. M., y Lucas, R. E. (2006). The Mini-IPIP Scales: Tiny-yet-effective measures of the Big Five Factors of Personality. Psychological Assessment, 18, 192-203.

Green-Demers, I., Pelletier, L., y Ménard, S. (1997). The impact of behavioural difficulty on the saliency of the association between self-determined motivation and environmental behaviours. Canadian Journal of Behavioural Science/Revue Canadienne des Sciences du Comportement, 29, 157-166.

Goldberg, L. R. (1999). A broad-bandwidth, public-domain, personality inventory measuring the lower-level facets of several five-factor models. En I. Mervielde, I. J. Deary, F. De Fruyt, yF. Ostendorf (Eds.), Personality Psychology in Europe (Vol. 7, pp. 7-28). Tilburg University Press.

Hirsh, J. B. (2010). Personality and environmental concern. Journal of Environmental Psychology, 30, 245-248. 
Hirsh, J. B., y Dolderman, D. (2007). Personality predictors of consumerism and environmentalism: A preliminary study. Personality and Individual Differences, 43, 1583-1593.

Huta, V. (2013). Pursuing Eudaimonia versus Hedonia: Distinctions, similarities, and relationships. En A. S. Waterman (Ed.), The best within us: Positive psychology perspectives on eudaimonia (pp. 139-158). American Psychological Association.

Iwata, O. (2002). Coping Style and Three Psychological Measures Associated with Environmentally Responsible Behavior. Social Behavior and Personality, 30, 661669.

Lyubomirsky, S., y Lepper, H. (1999). A measure of subjective happiness: Preliminary reliability and construct validation. Social Indicators Research, 46, 137-155.

Manríquez, J. C., Corral, V., y Vanegas, C. (2016). Positive (gratitude, eudaimonia) and negative (scarcity, costs) determinants of water conservation behavior. Psyecology, 7, 178-200.

Markowitz, E., Goldberg, L., Ashton, M., y Lee, K. (2012). Profiling the 'proenvironmental individual': A personality perspective. Journal of Personality, 80, 81-111.

Moll, J., Krueger, F., Zahn, R., Pardini, M., Oliveira, R., y Grafman, J. (2006). Human fronto-mesolimbic networks guide decisions about charitable donation. Proceedings of the
National academy of Sciences of the United States of America, 103, 15623-15628.

Moore, H., y Boldero, J. (2017). Designing interventions that last: a classification of environmental behaviors in relation to the activities, costs, and effort involved for adoption and maintenance. Frontiers in Psychology, 8, 1874.

Peterson, C., Park, N., y Seligman, M. E. (2005). Orientations to happiness and life satisfaction: The full life versus the empty life. Journal of Happiness Studies, 6, 25-41.

Ryff, C. D. (1989). Happiness is everything, or is it? Explorations on the meaning of psychological well-being. Journal of Personality and Social Psychology, 57, 1069-1081.

Sauer, P., y Dick, A. (1993). Using moderator variables in structural equation models. Advances in Consumer Research, 20, 636640.

Steg, L., Perlaviciute, G., van der Werff, E., y Lurvink, J. (2014). The significance of hedonic values for environmentally relevant attitudes, preferences, and actions. Environment and Behavior, 46, 163-192.

Swim, J., Clayton, S., y Howard, G. (2011). Human behavioral contributions to climate change. Psychological and contextual drivers. American Psychologist, 66, 251-264. Tapia, C., Corral, V., Fraijo, B., y Durón, F. (2013). Assessing sustainable behavior and its correlates: a measure of pro-ecological, 
frugal, altruistic and equitable actions. Sustainability, 5, 711-723.

Toegel, G., y Barsoux, J. L. (2012). How to become a better leader. MIT Sloan Management Review, 53, 51-60.

Vanegas-Rico, M. C., y Bustos, M. (2019).

Relación del costo personal y creencias ambientales con compras verdes. Nthe, 26, 1-6.

Vanegas-Rico, M. C., y Corral-Verdugo, V. (2015). La percepción del costo conductual en el comportamiento proambiental. Memorias del XXIII Congreso Mexicano de Psicología. (pp. 1616-1617). Sociedad Mexicana de Psicología.

Verhallen, T. M. M., y Pieters, R. (1984). Attitude theory and behavioral costs. Journal of Economic Psychology, 5, 223-249.

Waterman, A. S., Schwartz, S., y Conti, R. (2008). The implications of two conceptions of happiness (hedonic enjoyment and eudaimonia) for the understanding of intrinsic motivation. Journal of Happiness Studies, 9, 41-79.

Enviado: 21/01/2020

Revisado: $10 / 02 / 2020$

Aceptado: 27/05/2020 